\title{
Synthesis of 3D porous carbon based on cheap polymers and graphene foam for
}

\section{high-performance electrochemical capacitors}

Farshad Barzegar ${ }^{a}$, Abdulhakeem Bello $^{a}$, Omobosede O Fashedemi $^{b}$, Julien K. Dangbegnon ${ }^{a}$, Damilola Y. Momodu ${ }^{a}$, Fatemeh Taghizadeh ${ }^{a}$, and Ncholu Manyala ${ }^{a^{*}}$

${ }^{a}$ Department of Physics, Institute of Applied Materials, SARCHI Chair in Carbon Technology and Materials, University of Pretoria, Pretoria 0028, South Africa.

${ }^{b}$ Department of Chemistry, University of Pretoria, Pretoria 0002, South Africa.

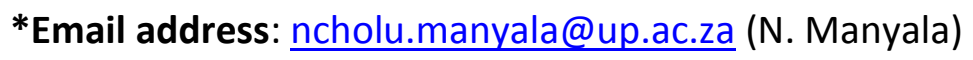

Tel: +27 (0)12420 3549, Fax: +27 (0)124202516

\section{ABSTRACT}

A scalable production of high surface area nanoporous carbon material $\left(\sim 2994 \mathrm{~m}^{2} \mathrm{~g}^{-1}\right)$ with good distribution of micro-, meso- and macro-pores was hydrothermally synthesized using both cheap polymers and graphene foam as carbon sources. The as synthesised material shows a unique interconnected porous graphitic structure. The electrochemical double-layer capacitor fabricated from this nanoporous carbon material exhibited a superior supercapacitive performance of $188 \mathrm{~F} \mathrm{~g}^{-1}$ at current density $0.5 \mathrm{~A} \mathrm{~g}^{-1}$. This corresponded to areal capacitance of $6.3 \mu \mathrm{F} \mathrm{cm}{ }^{-2}$ coupled with a high energy of $0.56 \mu \mathrm{Wh} \mathrm{cm}{ }^{-2}\left(16.71 \mathrm{Wh} \mathrm{kg}^{-1}\right)$ and a power density of 13.39 $\mu \mathrm{W} \mathrm{cm}$ (401 $\mathrm{W} \mathrm{kg}^{-1}$ ) due to extended potential window of $1.6 \mathrm{~V}$ in $\mathrm{KOH}$ aqueous electrolyte. Moreover, no capacitance loss after 10,000 cycles was observed, owing to the 
unique structure and large surface area of the active material. The outstanding performance of this material as supercapacitor electrode shows that it has great potential for high performance energy-related applications.

KEYWORDS: Porous carbon; Supercapacitor; Equivalent circuit; Electrochemical performance.

\section{GRAPHICAL ABSTRACT \\ GF $\mathrm{HCl}$ \\ PVA $7^{+}+\frac{1}{+}$ PVP}
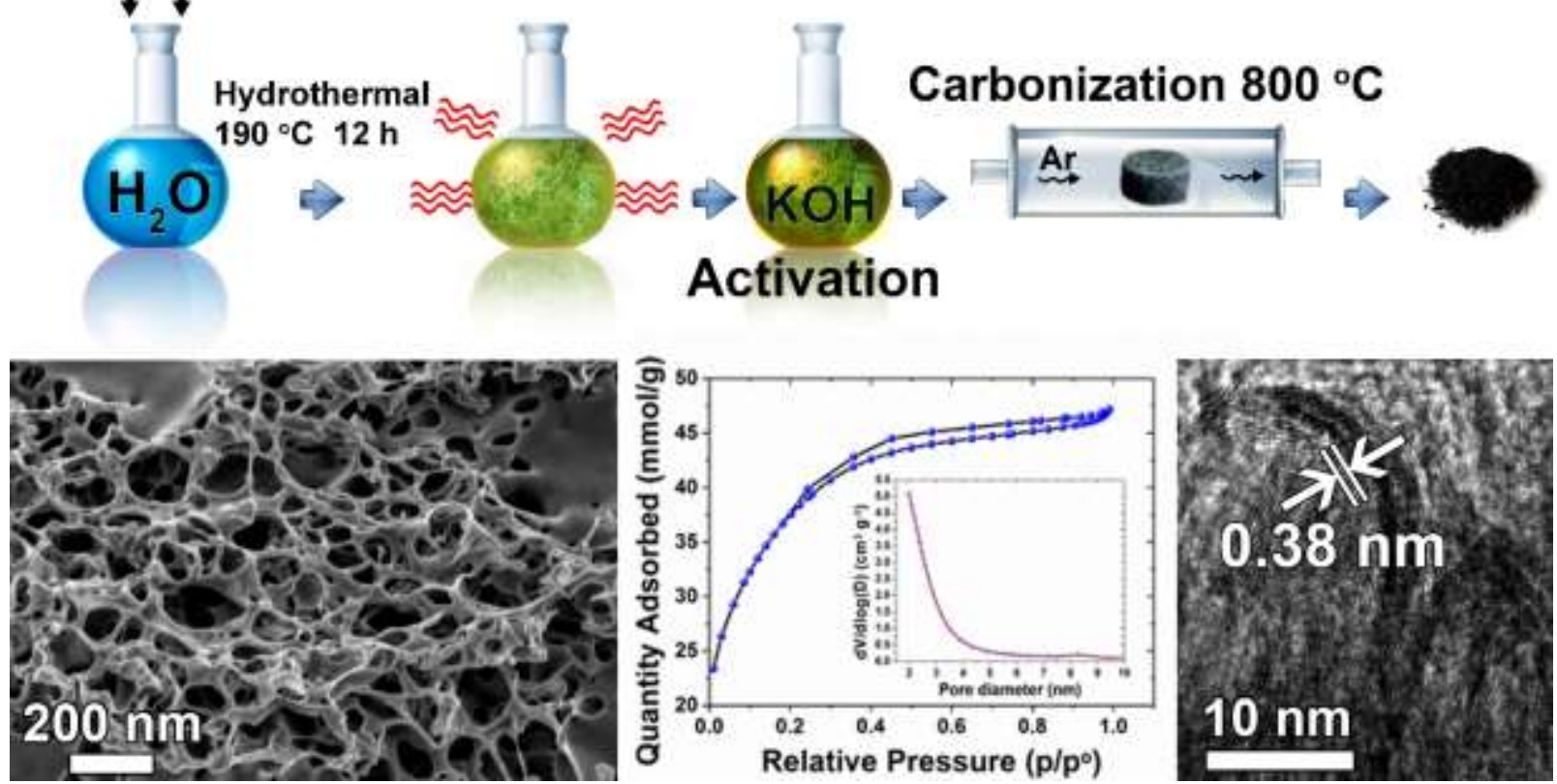

\section{INTRODUCTION}

As a result of the depletion of fossil fuels and the issue of greenhouse gas emission, cleaner sources of energy are being explored such as solar and wind energies. Furthermore, new technologies for minimizing $\mathrm{CO}_{2}$ emission are developing rapidly and require the elaboration of highly reliable energy storage devices. Supercapacitors or electrical double layer capacitors (EDLCS) seem to be the most likely candidates for the next generation of energy storage devices. They are at the forefront of research on energy storage technology due to the 
limitations of batteries for many applications. They can help to optimize energy consumption by complementing batteries in a variety of applications. They are also regarded as possible replacements for batteries if their production costs are competitive enough and their energy densities surpass those of current batteries. At the same time, they must be able to retain their extraordinary properties of high power density, superior rate capability, rapid charging/discharging rate, long cycle life $(>100,000$ cycles), simple principles, fast dynamics of charge propagation and low maintenance cost. These unique properties result from the storage harvesting mechanism of the primary charge, which relies on the electro-sorption of ions from an electrolyte onto a porous electrode [1].

Carbon materials are the materials of choice for EDLC applications. Their advantages as electrode materials were reported in previous work [2] some of which are the following: carbon has different allotropes, a variety of shapes, and good polarization, and its amphoteric behavior exhibits essential electrochemical properties from donor to acceptor states. It is also environmentally friendly. However, although low-cost nanoporous carbon electrodes with a tunable electrically conductive architecture and a highly active surface area exhibit high power density above $10 \mathrm{~kW} \mathrm{~kg}^{-1}$ their energy density is still limited to less than $10 \mathrm{Wh} \mathrm{kg}^{-1}$ [3]. Even though the operating voltage can be up to $4 \mathrm{~V}$ for ionic liquids (ILs) electrolytes for carbon electrodes, the ILs electrolytes are usually characterized by poor conductivity and high viscosity which significantly hinder their penetration in the electrode structure and decreases charge propagation which results in low energy density. Consequently, the capacitance of the capacitors operating in such electrolytes is usually low and is characterized by poor power rates [4]. On the other hand, aqueous electrolytes are cheap and ecological but suffer from the 
drawback of even smaller operating voltage as low as $1.0 \mathrm{~V}$ due to the thermodynamic decomposition of water at $1.23 \mathrm{~V}$. However, this electrolyte has the highest specific capacitance compared to organic and ionic electrolytes owing to its high conductivity, low viscosity and good charge propagation. In addition, this electrolyte is environmentally friendly which solves the problem of disposal of waste and used devices. Moreover, functional carbon materials or carbon materials enriched with heteroatom such as oxygen and nitrogen, demonstrate pseudocapacitance originating from redox reactions of these functional groups as well as from local changes in the electron density of the carbon matrix enriched by these heteroatoms. These functionalized or enriched carbon materials are more stable in an aqueous electrolyte, and their pseudocapacitive behavior further increases their specific capacitance. These advantages have encouraged research into producing novel designs that could increase the working potential of EDLCs in aqueous electrolytes close to that of organic electrolytes (i.e. 2 V). Recently Fic et al. [5] designed an activated carbon (AC) symmetric supercapacitor exhibiting

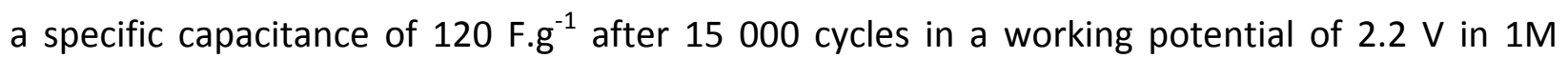
$\mathrm{Li}_{2} \mathrm{SO}_{4}$ aqueous electrolyte. Furthermore, novel asymmetric supercapacitors based on carbon in $1 \mathrm{M} \mathrm{H}_{2} \mathrm{SO}_{4}$ have demonstrated high stability after 10,000 cycles at $1.6 \mathrm{~V}$ [6]. The high overpotential of di-hydrogen evolution was used to improve the working voltage. These two reports were based on commercial activated carbon with a high specific surface area (SSA) but they gave no information on the pore sizes distribution (PSD). In fact, good PSD in the carbon materials can enhance the specific capacitance of the EDLCs since sub-nanometric pores size between 0.7 and $0.9 \mathrm{~nm}$ satisfy the ions electro-sorption. However mono-dispersion of these micropores adversely affects rapid transport of electrolyte ions by increasing the ion transport 
resistance in these small pores which leads to poor rate capability and power density. Therefore, the presence of mesopores and macropores is essential for ions transport and the ion buffering reservoir respectively [7]. Carbide derived carbons (CDCs), usually produced from chlorination of metal carbides, possess narrow and tunable PSDs between 0.6 and $2 \mathrm{~nm}$ and a high SSA up to $3000 \mathrm{~m}^{2} \mathrm{~g}^{-1}$. These properties can be controlled and tailored by the selection of the precursors and the chlorination conditions. These materials show extraordinary electrochemical performances in any electrolyte [8]. However, CDCs are very expensive and serious safety and environmental issues surrounding their production are limiting factors for their commercialization. On the other hand, polymers have been actively investigated as promising alternative precursors. They have received considerable interests in recent years due to their low cost, commercial availability, uniform structure, and high chemical purity. Mixed with graphene, they can act as spacers to form composites, preventing graphene agglomeration or re-stacking [9]. Thus, the high surface area of graphene will be accessible to ions and its high conductivity will also be advantageous for the performance of this graphene-based material. Furthermore, there have been previous reports on carbon derived hydrogel for various applications. For example, You et al. [10] prepared a hydrogel derived non-precious electrocatalysts for efficient oxygen reduction reaction (ORR) by freeze-drying and subsequent carbonization of a Tris/Boric acid/EDTA gel. In their more recent work [11], similar synthesis technique was used to produce multifunctional electroactive heteroatom-doped carbon aerogels. This material showed promising results when used as active material for ORR and lithium ion storage. Zhang et al [12] synthesized N-doped carbon aerogels with high surface 
area. This electrode material demonstrated good electrochemical capacitor performance with a specific capacitance of $354 \mathrm{Fg}^{-1}$ at a current density of $0.2 \mathrm{Ag}^{-1}$.

In this paper, we report on the hydrothermal synthesis of three dimensional porous carbons with a high SSA based on graphene foam (GF) with two different polymers namely Polyvinylpyrrolidone (PVP) and polyvinyl alcohol (PVA). We chose PVA and PVP as they are wellknown polymer blends [13-15]. PVA has some outstanding physical properties which include its electrochemical stability, non-toxicity and mechanical strength. PVP is also non-toxic and has excellent absorption and complex forming capabilities [16]. While PVA with its free OH groups attached to methane carbons can be a source of hydrogen bonding, PVP being a vinyl polymer with a pyrolidine group is good at attracting other polar groups and forming other bonds. Thus a highly cross-linked polymer hydrogel with ionic bonds, inter and intra molecular hydrogen bonds and dipole interactions is easily formed with such blends. The hydrothermal treatment resulted in hydrogels that were further treated with $\mathrm{KOH}$ for activation followed by carbonization to create a porous network of graphitic carbons. A systematic study was made of the effect of activation on the morphology and SSA of the three-dimensional PVA-GF-PVP produced carbons designated as 3D-PGP. The electrochemical characteristics of the 3D-PGP were investigated using both the two- and three- electrode configurations. The 3D-PGP displayed remarkable electrochemical properties in a conventional $6 \mathrm{M} \mathrm{KOH}$ aqueous electrolyte. 


\section{EXPERIMENTAL}

\subsection{Synthesis and activation of the hydrogel.}

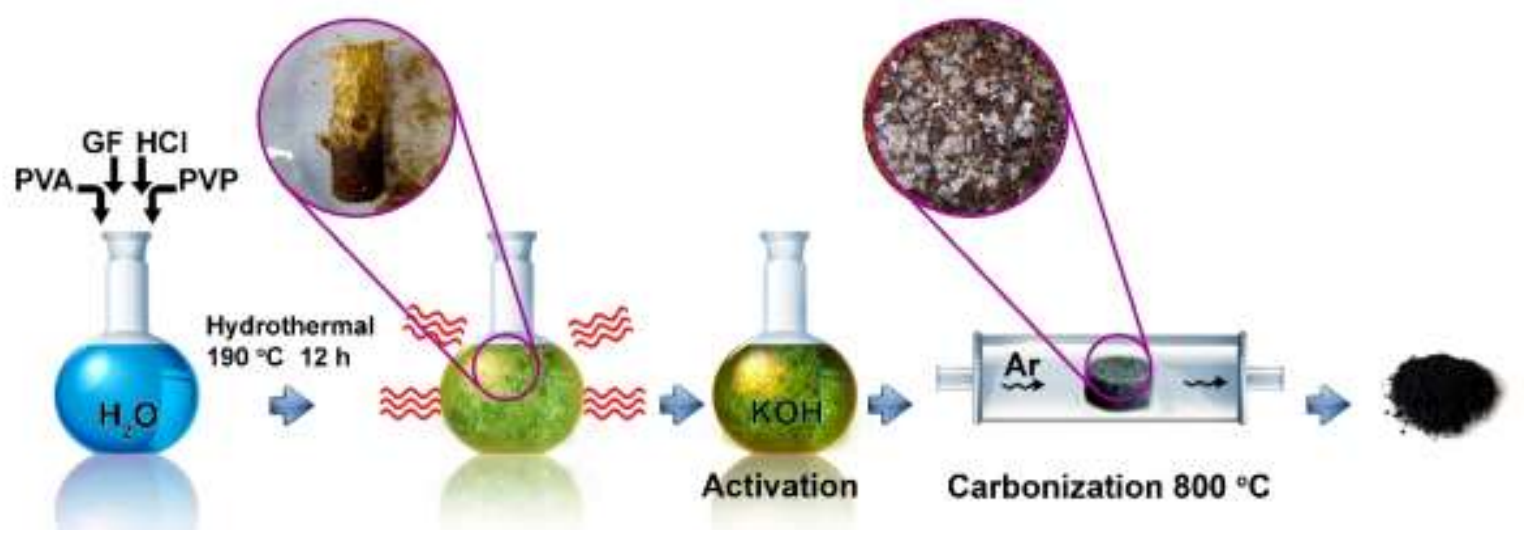

Scheme 1. Schematic diagram of the preparation process of 3D-PGP carbons.

Scheme 1 presents the preparation process of carbons. Graphene foam (GF) with a three dimensional network and relatively low defect density (Figure S1 (a)) was first prepared by chemical vapor deposition (CVD) following the procedure used in our previous work [17]. The hydrogel was synthesised via the hydrothermal process. Typically, different masses of GF were dispersed in $100 \mathrm{ml}$ of water containing $11.11 \mathrm{~g}$ of polyvinyl alcohol (PVA) followed by the addition of different masses of Polyvinylpyrrolidone (PVP) (for PVA/GF/PVP samples) in a glass vial by ultrasonication, $1.50 \mathrm{ml}$ of hydrochloric acid $(\mathrm{HCl})$ was added to the solution as a cross linker [18]. The mixture was sonicated for few minutes and further stirred for 30 min to obtain a homogeneous dispersion before being transferred into a $120 \mathrm{ml}$ Teflon-lined autoclave system operated at $190{ }^{\circ} \mathrm{C}$ for $12 \mathrm{~h}$. The resulting hydrogel was crushed and washed with deionized water and dried for $6 \mathrm{~h}$. The hydrogel obtained was then soaked in aqueous $\mathrm{KOH}$ solution with a $\mathrm{KOH} /$ hydrogel mass ratio of 5 for $24 \mathrm{~h}$ and dried at $120{ }^{\circ} \mathrm{C}$ for $12 \mathrm{~h}$ before 
carbonization [19]. The composite was then placed in a horizontal tube furnace ramped from room temperature to $800^{\circ} \mathrm{C}$ at $10^{\circ} \mathrm{C} / \mathrm{min}$ under argon gas flow for $1 \mathrm{~h}$ of activation. This procedure transforms the hydrogel into carbon materials consisting of a continuous pore network distribution. After which samples were washed with $3 \mathrm{M} \mathrm{HCl}$ to remove the remaining $\mathrm{KOH}$ and then washed with deionized water. Samples were designated 3D-PG-1 when 50 mg of GF was added, 3D-PG-2 when $80 \mathrm{mg}$ of GF was added, and 3D-PG-3 when 100mg of GF was added in a PVA solution. 3D-PGP-1 denotes the addition of $1.76 \mathrm{~g}$ PVP sample to 3D-PG-3, and 3D-PGP-2 indicates samples produced when $3.5 \mathrm{~g}$ of PVP was added to sample 3D-PG-3.

\subsection{Structural characterization}

The SEM images were obtained using a Zeiss Ultra Plus 55 field emission scanning electron microscope (FE-SEM) operated at an accelerating voltage of $2.0 \mathrm{kV}$. The energy dispersive x-ray (EDX) obtained using scanning electron microscope (SEM) (JEOL 5800LV) equipped with an EDX spectrometer operated at $20 \mathrm{kV}$ was used to estimate the elemental composition of the produced materials. X-ray photoelectron spectroscopy (XPS) was used to determine the chemistries of activated carbons obtained in powder form. A Physical Electronics VersaProbe 5000 instrument employing a $100 \mu \mathrm{m}$ monochromatic Al-K $\alpha$ to irradiate the sample surface was used. Photoelectrons were collected by a $180^{\circ}$ hemispherical electron energy analyzer. Samples were analyzed at a $45^{\circ}$ angle between the sample surface and the path to the analyzer. Survey spectra were taken at a pass energy of $117.5 \mathrm{eV}$, with a step size of $0.1 \mathrm{eV}$, which was used to obtain an estimate of the elemental analysis of the powders. High-resolution spectra of C1s, $\mathrm{N} 1 \mathrm{~s}$, and $\mathrm{O} 1 \mathrm{~s}$ regions were taken at a pass energy of $23.5 \mathrm{eV}$, with a step size of $0.05 \mathrm{eV}$. All 
binding energies were referenced to that of the binding energy of C-C at $284.7 \mathrm{eV}$. Transmission electron microscopy (TEM) was carried out with a JEOL JEM-2100F microscope operated at 200 kV accelerating voltage. Nitrogen adsorption-desorption isotherms were measured at $-196{ }^{\circ} \mathrm{C}$ using a Micromeritics ASAP 2020. All the samples were degassed at $180^{\circ} \mathrm{C}$ for more than $12 \mathrm{~h}$ under high vacuum conditions. The surface area was calculated by the Brunauer-EmmettTeller (BET) method from the adsorption branch in the relative pressure range $\left(\mathrm{P} / \mathrm{P}_{0}\right)$ of 0.01 0.2. The X-ray diffraction (XRD) was recorded using an XPERT-PRO diffractometer (PANalytical BV, the Netherlands) with theta/theta geometry. The qualitative phase of samples was analyzed with the X'pert Highscore search match software at room temperature. Raman spectroscopy measurements were carried out using a Jobin Yvon Horiba TX 6400 micro-Raman spectrometer equipped with a triple monochromator system to eliminate contributions from Rayleigh lines. LabSpec (Ver. 5.78.24) analytical software was used to analyze the spectra. All the samples were excited with a $514 \mathrm{~nm}$ argon laser line with a laser power of $1.5 \mathrm{~mW}$ to avoid possible thermal effects.

\subsection{Electrochemical testing}

The electrodes for supercapacitor were prepared as follows: The synthesized carbon material was mixed with Polyvinylidene fluoride (PVdF), with a mass ratio of 90:10. The slurry was made using N-methylpyrrolidone (NMP) solution and was uniformly coated on nickel foam acting as current collector. The electrodes were dried at $60^{\circ} \mathrm{C}$ in an oven for 8 hours to ensure complete evaporation of the NMP. The electrochemical test of the symmetric cell was carried out in a two-electrode cell configuration with coin-type cells. Both electrodes had a mass loading of 
$\sim 3.3 \mathrm{mg}$ with a thickness of $0.2 \mathrm{~mm}$ and a diameter of $16 \mathrm{~mm}$. Glass microfiber filter paper was used as the separator in $6 \mathrm{M} \mathrm{KOH}$ aqueous electrolyte solution. The three-electrode measurement was performed with 3D-PGP-1 as the working electrode, a glassy carbon plate as the counter electrode and $\mathrm{Ag} / \mathrm{AgCl}(3 \mathrm{M} \mathrm{KCl})$ as the reference. All electrochemical measurements were carried out using a Bio-logic SP-300 potentiostat namely cyclic voltammetry (CV), chronopotentiometry (CP) and electrochemical impedance spectroscopy (EIS). The $\mathrm{CV}$ tests were carried out in the potential range of 0 to $1.6 \mathrm{~V}$ at different scan rates ranging from $5 \mathrm{mV} \mathrm{s}^{-1}$ to $100 \mathrm{mV} \mathrm{s}^{-1}$. Electrochemical impedance spectroscopy (EIS) measurements were conducted in the frequency range from $0.1 \mathrm{~Hz}$ to $100 \mathrm{kHz}$ with an open circuit potential ( 0 V).

According to the galvanostatic charge-discharge tests based on a two-electrode cell, the gravimetric specific capacitance $\left(C_{s p}: F g^{-1}\right)$, the maximum energy density $\left(E_{\max }: W h \mathrm{~kg}^{-1}\right)$, and the power density $\left(P_{\max }: \mathrm{kW} \mathrm{kg}^{-1}\right)$ of the cell were calculated using equations 1 to 3 respectively: $\mathrm{C}_{\mathrm{sp}}=4 \times \mathrm{I} \Delta \mathrm{t} / \mathrm{m} \Delta \mathrm{V}$

$\mathrm{E}_{\max }=0.5 \mathrm{C}(\Delta \mathrm{V})^{2}=\left(\mathrm{C}_{\mathrm{sp}} \times \Delta \mathrm{V}^{2}\right) / 28.8$

$\mathrm{P}_{\max }=3.6 \times \mathrm{E}_{\max } / \Delta \mathrm{t}$

where $I$ is the discharge current $(A), m$ is the total mass of the active material in both electrodes (g), $\Delta \mathrm{t}$ is the discharge time $(\mathrm{s})$, and $\Delta \mathrm{V}$ is the applied potential $(\mathrm{V})$. The areal $\left(\mathrm{C}_{\text {area }}: \mathrm{F} \mathrm{cm}^{-2}\right)$ capacitances of 3D-PGP were calculated using equation 4:

$\mathrm{C}_{\text {area }}=10^{-4} \times \mathrm{C}_{\mathrm{sp}} / \mathrm{A}_{\mathrm{BET}}$ 
where $A_{B E T}$ is the BET surface area $\left(\mathrm{m}^{2} \mathrm{~g}^{-1}\right)$ of the 3D-PGP-1 sample. The energy $\left(E_{a}: W h \mathrm{~cm}^{-2}\right)$ and power $\left(\mathrm{P}_{\mathrm{a}}: \mathrm{kW} \mathrm{cm}{ }^{-2}\right)$ densities of the two electrode cell were calculated respectively using equation 5 and 6 below respectively:

$\mathrm{E}_{\mathrm{a}}=\left(\mathrm{C}_{\text {area }} \times \Delta \mathrm{V}^{2}\right) / 28.8$

$\mathrm{P}_{\mathrm{a}}=3.6 \times \mathrm{E}_{\mathrm{a}} / \Delta \mathrm{t}$

\section{RESULTS AND DISCUSSION}

Figure 1 shows SEM and TEM micrographs of 3D-PGP-1 sample. SEM images of GF, 3D-PGP-2 and 3D-PGP-3 samples are shown in figure S1, in the supporting information. Figure 1 (a) shows a low-magnification image of 3D-PGP-1 sample, and Figure 1 (b) shows a high-magnification image of the sample, which reveals a highly dense and interconnected porous architecture which is beneficial in providing a large ion-accessible surface for fast ion transport for high performance supercapacitors. The TEM image (Figure 1 (c)) verifies that the pores are homogeneously and uniformly distributed throughout the highly porous structure of the sample with a d-spacing of about $0.38 \mathrm{~nm}$ as indicated by the distance between the white lines in the TEM image. 

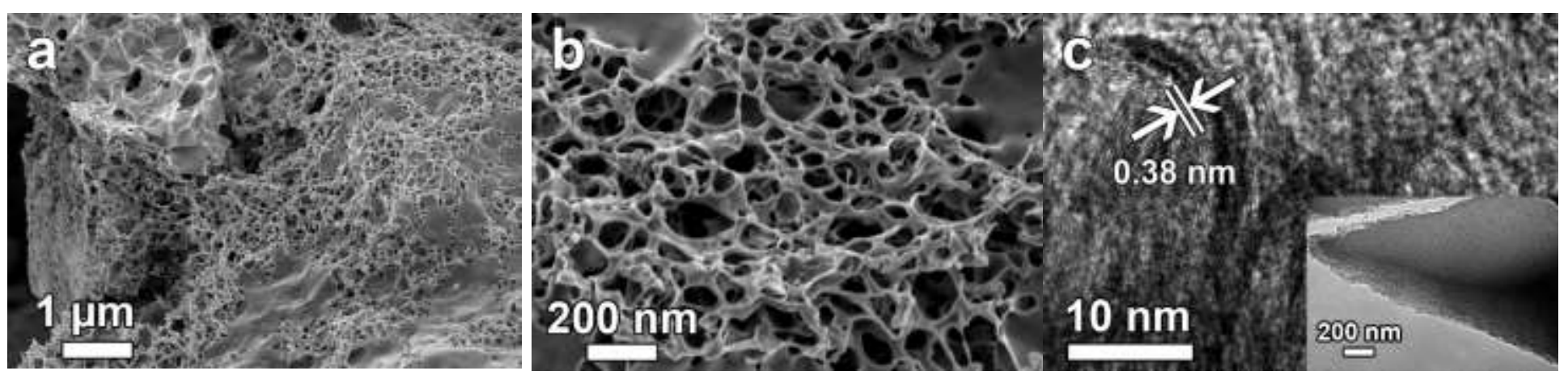

Figure 1. SEM and TEM of 3D-PGP-1 sample: (a) low-magnification SEM image, (b) high-magnification SEM image and (c) TEM image.

The mechanism of formation of the hydrogel which yielded the porous structure of 3D-PGP-1 sample could be explained as follows: The PVA, which is the template, consists of long molecular chains which are believed to promote physical cross-linking points with the PVP. The PVP promotes and ensures a high cross-linking density and denser packing of polymer chains in the PVP/PVA hydrogel network [20]. The deprotonating processes in the acid catalyzing medium enhances the formation of bonds between the two polymers. The hydroxyl OH groups of PVA readily deprotonate in an acidic medium, making a cross-link with carbonyl groups (CO) of PVP via hydrogen bonding [14]. Another form of cross linkage between the two polymers can occur as a result of free radicals produced at the main chains of both polymers. This can also be enhanced during the heating of the polymer mixtures. Thus other bonds can be formed via the free radicals of the PVP groups and the OH of PVA (as can be seen below in reaction Scheme 2) 

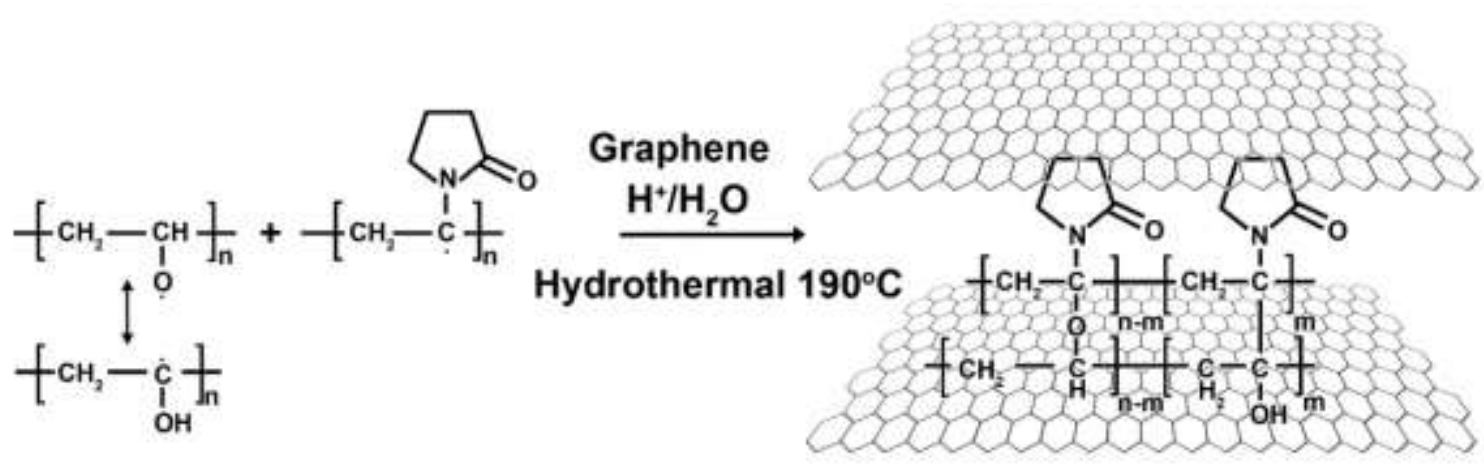

Scheme 2. Schematic of the reaction between PVA/GF/PVP in a hydrothermal system.

below leading to more cross-linking. This cross-linkage further assists in the interconnectivity and the porosity of the structure. The different repeating units in PVA and PVP also result in a steric stabilization of the hydrogel produced [21].

On the other hand the presence of graphene foam which has been shown to have excellent properties by Chen et. al. [22], is believed to have a $\pi-\pi$ or weak Van der Waal interaction with the hydrogel, which ensures a larger surface and enhanced conductivity. The activation of the preformed PVA/GF/PVP hydrogel by $\mathrm{KOH}$ further enhances the porosity of the hydrogel structure by the efficient intercalation of potassium ions in the carbon lattice of the polymer network. The role of $\mathrm{KOH}$ in the activation of carbon materials has been summarized as the result of a series of synergistic reactions, which include chemical activation, physical activation and carbon lattice expansion by the metallic $\mathrm{K}$ intercalation [23]. The resulting activated carbon obtained from the PVA/GF/PVP hydrogel after the carbonation process through annealing thus guaranteed a highly dense porosity which should be coupled with a highly improved conductive surface area. 
The XPS elemental analysis and peak fitting results are shown in table S1 and S2 respectively in the supporting information. Carbon is considered the major element ( 89.4 at.\%) in the sample, while oxygen takes the second place, the percentage of oxygen is 9.5 at. \%. The sample also contains $\mathrm{N}$ at 1.1 at. \%. The results of the peak fitting for the sample are shown in Figure 2 . The C1s region was fitted by four components corresponding to graphitic carbon, hydrocarbons $\left(\mathrm{CH}_{\mathrm{x}}\right)$, alcoholic (C-O-) and/or carbon nitrogen structures (C-N-), and carboxyl or ester (COO). The graphitic carbon takes the largest fraction of the C1s region (0.45). The N1s region (Figure.
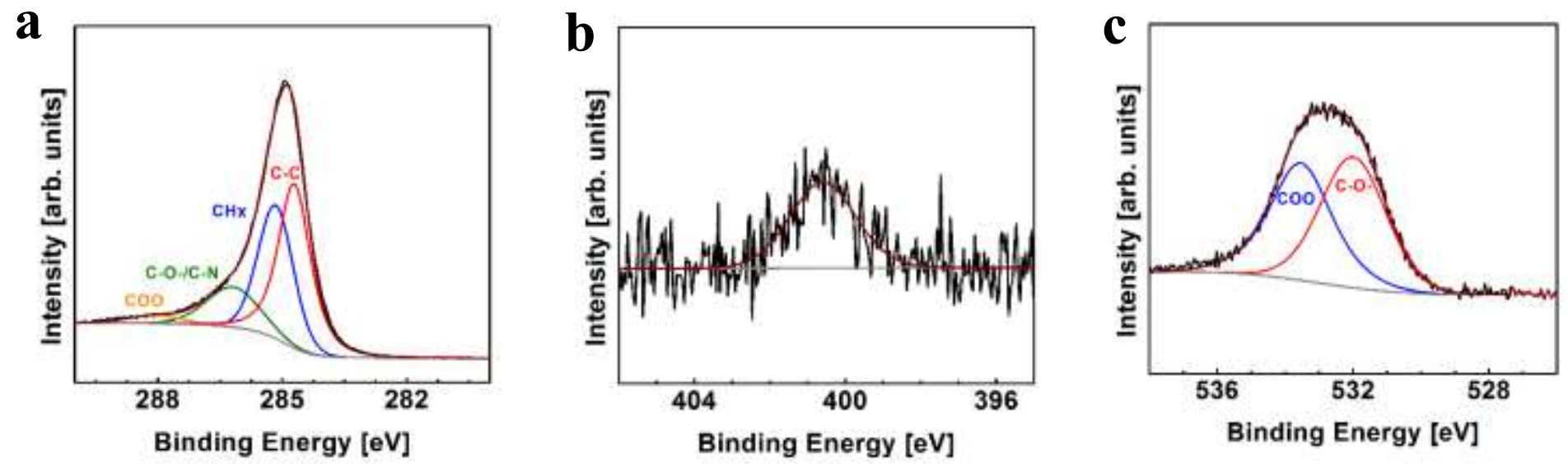

Figure 2. XPS spectra of (a) C1s, (b) N1s and (c) O1s regions for 3D-PGP-1.

2b) was fitted by one component corresponding to nitrile and/or pyrrole while the O1s region (Figure 2c) was fitted by two components corresponding to $\mathrm{C}-\mathrm{O}-$ and $\mathrm{COO}$. The presence of all these functional groups on the surface of this activated carbon could prevent oxygen and hydrogen overpotential in aqueous electrolytes as mentioned by Bichat et. al. [24]. 
$\mathbf{a}$

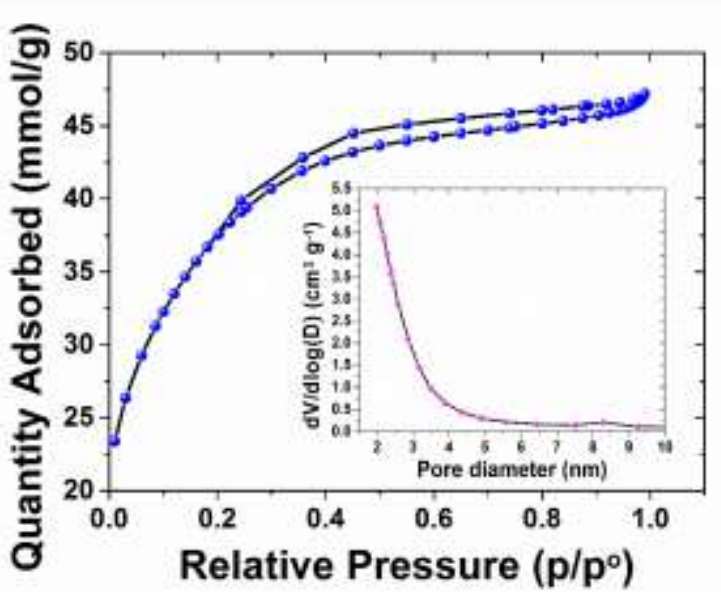

b

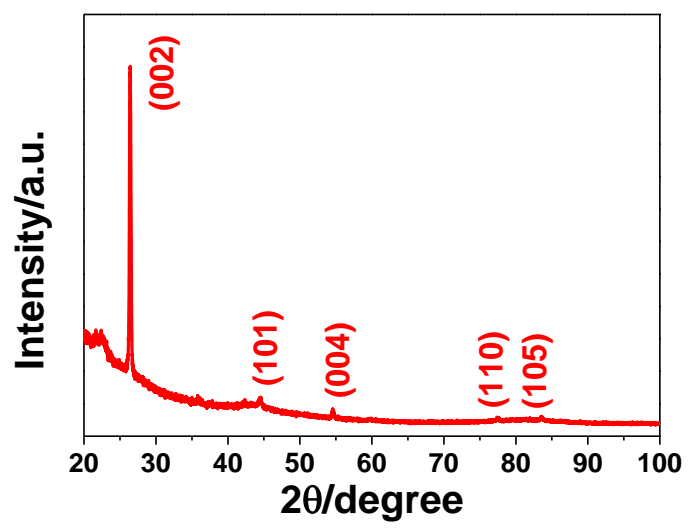

Figure 3. (a) $\mathrm{N}_{2}$ adsorption-desorption isotherm of the 3D-PGP-1 sample and (b) X-ray diffraction pattern of the 3DPGP-1 sample.

The specific surface areas and pore size distributions of samples were measured by $\mathrm{N}_{2}$ adsorption-desorption isotherms using the Barrett Joyner Halenda (BJH) method as shown in Figure 3 for the 3D-PGP-1 sample, with other samples showing similar behaviour. It is observed that the 3D-PGP-1 sample shows a H4 isotherm that is characteristic of complex materials that contain micropores and mesopores. At high pressure, the inflection point occurs near completion of the first monolayer. The sample also exhibits a high Brunauer-Emmett-Teller surface area of $2994 \mathrm{~m}^{2} \mathrm{~g}^{-1}$. The average BJH pore size distribution (inset to the figure) is of 2 $\mathrm{nm}$, with a substantial amount of mesopores present in the 3D-PGP-1 sample. The structural parameters, such as BET isotherm and pore volume of all the other samples are listed in Table S3 in the supporting information. Figure 3 (b) shows the XRD patterns for the 3D-PGP-1 powders. The wavelength used for the XRD analysis was $\mathrm{Cu}-\mathrm{K}_{\alpha}, 1.5405 \AA$. It is noted that all the XRD peaks are identified as graphite peaks (COD: 96-900-8570) which crystallize in the 
hexagonal structure with space group P63mc (186), and lattice parameters $\mathrm{a}=2.4560 \AA$ and $\mathrm{c}=$ 6.6960 A. Figure S2 is the EDX of the 3D-PGP-1, which shows the presence of carbon only.

Figure S3 in the supporting information shows the Raman spectra of all as-synthesized samples. Figure S3 (a) shows the defect-free spectrum of GF with only two prominent peaks (G and 2D peaks) typical of graphene samples. This shows that the CVD-grown GF consists of few layers. Figure S3 (b) shows the Raman spectra of the composites. Typically, all the Raman spectra show $D$ and $G$ bands, suggesting that the samples produced are made up of highly disordered graphitic structures. The $D$ band is commonly ascribed to a series of structural defects, whereas the $G$ band is observed in all graphitic structures. The intensity ratio of the $D$ - and G-peak $I_{D} / I_{G}$ is also used to estimate the concentration of $\mathrm{sp}^{3}$ carbon relative to the concentration of $\mathrm{sp}^{2}$ carbon.

The potential of the activated materials performance as an EDLC electrode was demonstrated using cyclic voltammetry (CV) and galvanostatic charge/discharge (CD) measurements in both three- and two- electrode cell configurations. Table S3 shows that the 3D-PGP-1 sample has superior listed properties as compared to the others, and hence based on this, it was the sample on which electrochemical measurements were done because its properties are ideal for such measurements. Herein, we show the results of the 3D-PGP-1 sample with SSA of 2,994 $\mathrm{m}^{2}$ $\mathrm{g}^{-1}$. Figure 4 (a) shows the $\mathrm{CV}$ results of the 3D-PGP-1 as the active electrode material at two 

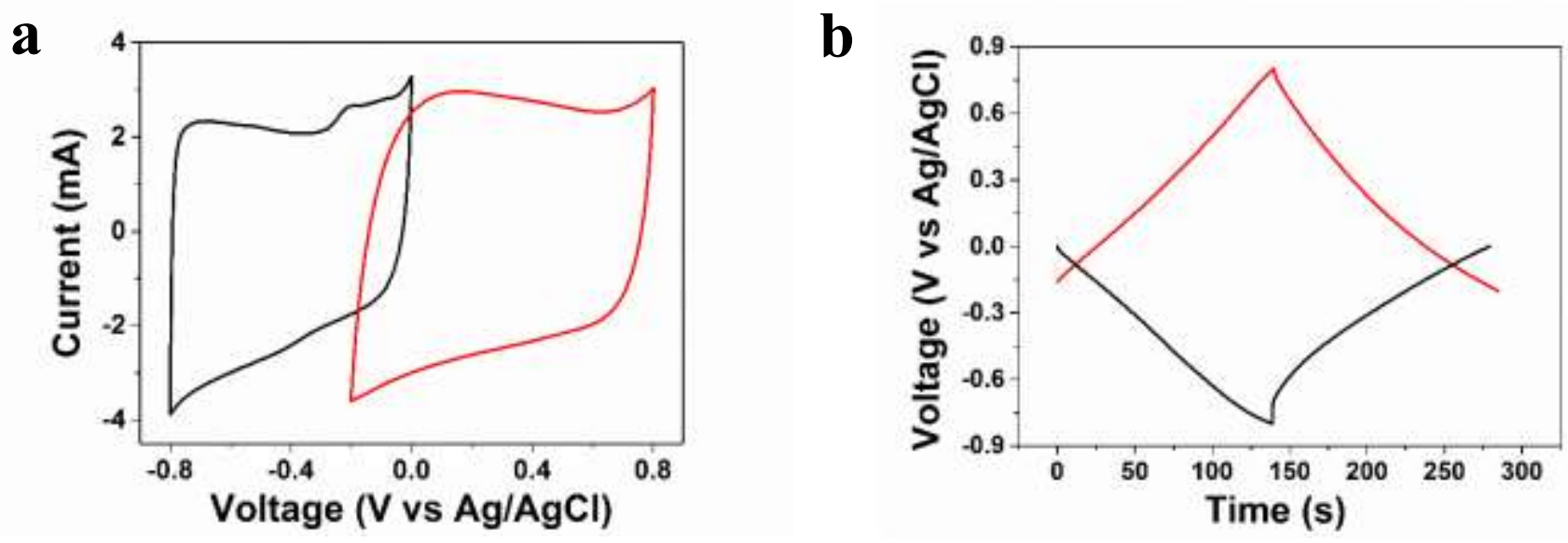

Figure 4. (a) CV and (b) galvanostatic charge/discharge results the 3D-PGP-1 sample in a three-electrode system in the negative and positive voltage ranges.

different voltage ranges of $-0.8-0 \mathrm{~V}$ and $-0.2-0.8 \mathrm{~V}$ vs $\mathrm{Ag} / \mathrm{AgCl}$. The $\mathrm{CV}$ curve in Figure 4 (a) is almost featureless and quasi-rectangular in these potential windows confirming the traditional double-layer capacitance characteristics. Figure 4 (b) shows the CD results of the 3D-PGP-1 sample in the three-electrode system; the same absolute values of the potential windows are shown. The specific capacitance values calculated from the CD in Figure 4 (b) are $175.27 \mathrm{~F} \mathrm{~g}^{-1}$ for the negative potential and $182.32 \mathrm{~F} \mathrm{~g}^{-1}$ for the positive potential. The variation in the values could be due to the distorted CV shape and possible increase in resistance as observed in the IR drop in the negative potential window. Due to these capacitance values, which might be related to the high SSA of this sample, further tests were done on a two-electrode symmetric configuration. 

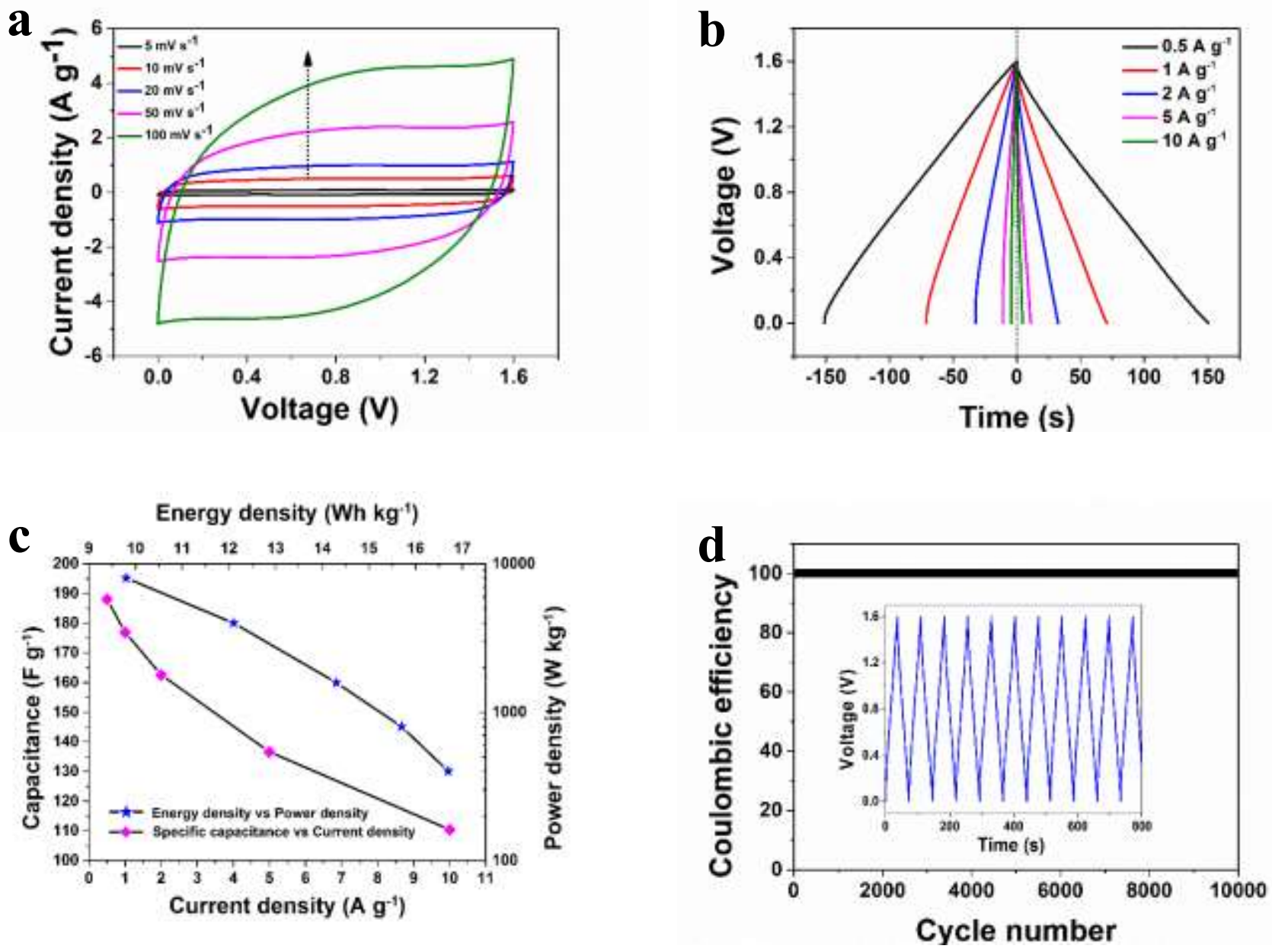

Figure 5. (a) CV curves at scan rates from 5 to $100 \mathrm{~m} \mathrm{Vs}^{-1}$, (b) The galvanostatic charge/discharge curves from 0.5 to $10 \mathrm{~A} \mathrm{~g}^{-1}$, (c) Ragone plot and the specific capacitance as function of the current density, and (d) Cycle stability at a constant current density of $2 \mathrm{~A} \mathrm{~g}^{-1}$.

Figure 5 presents the $\mathrm{CV}$ and Chronopotentiometry measurements of the symmetric $\mathrm{AC} / \mathrm{AC}$ supercapacitor in a $6 \mathrm{M} \mathrm{KOH}$ electrolyte. Figure 5 (a) shows the CV curves of the symmetric cell at different scan rates, which range from 5 to $100 \mathrm{mV} \mathrm{s}^{-1}$ in a potential window of $1.6 \mathrm{~V}$. The cell shows near-rectangular CV shapes at low scan rates, indicating ideal capacitive behavior, which demonstrates the formation of a double-layer capacitor and ideal polarization of the electrodes material. The shape of the CV indicates a pure capacitive characteristic behavior, suggesting 
reversible reaction at the electrode/electrolyte interface [25]. At a high scan rate, the CV shows a deviation from the pure EDLC shape with a characteristic resistive CV shape related to an increase in resistance attributed to the capacitance decay observed in Figure 5 (c). A symmetric triangular charge/discharge distinctive of ideal capacitor behavior was observed even at a high current density of $10 \mathrm{~A} \mathrm{~g}^{-1}$ (Figure 5 (b)). This linear behaviour indicates the reversible adsorption/desorption of ions at the electrode interface with a relatively small voltage drop. The gravimetric capacitance was $188 \mathrm{~F} \mathrm{~g}^{-1}$ at a current density of $0.5 \mathrm{~A} \mathrm{~g}^{-1}$, which can be expressed as areal capacitance from equation 4 as $6.28 \mu \mathrm{F} \mathrm{cm}^{-2}$. These are reasonable values for double-layer capacitors and compare well with or are higher than previous values in the literature $[5,26,27]$.

The Ragone plot and the specific capacitance as function of the current density of the symmetric device are shown in Figure 5 (c). The specific capacitance decreases from $188 \mathrm{~F} \mathrm{~g}^{-1}$ to $111 \mathrm{~F} \mathrm{~g}^{-1}$ with increasing current density from 0.5 to $10 \mathrm{~A} \mathrm{~g}^{-1}$, retaining about $60 \%$ of the initial capacitance. We attribute this to the unique nanoporous architecture, where the wide meso/macro pore range from $10-60 \mathrm{~nm}$ could buffer the amount of electrolyte ions and promote ion transfer into the interior pore surface of the micropores at higher current densities [28]. The maximum energy density of the device was recorded as $17 \mathrm{Wh} \mathrm{kg}^{-1}$ with power density of $400 \mathrm{~W} \mathrm{~kg}^{-1}$ at a current density of $0.5 \mathrm{~A} \mathrm{~g}^{-1}$. However, at this point it is worth stating that the gravimetric capacitance does not reflect the true performance metrics of the porous electrode materials [29] but rather the areal capacitance calculated according to equations 5 and 6. A high areal capacitance of $6.3 \mu \mathrm{F} \mathrm{cm}{ }^{-2}$ was obtained, which resulted in high energy and power densities of $0.56 \mu \mathrm{Wh} \mathrm{cm}{ }^{-2}\left(16.71 \mathrm{Wh} \mathrm{kg}^{-1}\right)$ and $13.39 \mu \mathrm{W} \mathrm{cm}{ }^{-2}\left(401 \mathrm{~W} \mathrm{~kg}^{-1}\right)$ respectively. 
This excellent electrochemical performance of the 3D-PGP-1 electrode material was attributed to: (i) The ability of the material to work in both the negative and positive potential window, thus extending the total potential of the fabricated cell; (ii) the unique porous architecture of the 3D-PGP-1 material and (iii) the large accessible surface area, which consists of micropores for trapping the ions and adequate proper-sized mesopores, which enable rapid and reversible ion transport [30]. Although, it is difficult to compare accurately the performance of all types of supercapacitors due to a large number of varying parameters such as material mass loadings and testing configurations, but a rough estimation and comparison show that the results presented in this work are good or even better than those in some previous reports on symmetric carbons devices in aqueous media $[26,31]$.

As shown in Figure 5 (d), the symmetric cell shows no capacitance loss after 10,000 cycles at a current density of $2 \mathrm{~A} \mathrm{~g}^{-1}$, demonstrating that repeated charge/discharge cycles never caused a significant structural change in the 3D-PGP-1 electrode. Nevertheless, a small increase in the capacitance ( $0.1 \%$ ) was observed after the cycling process. A similar increase was observed by Ren et .al [32] which was tentatively attributed to the swelling of the graphene at some defective sites. This promoted the intercalation of electrolyte ions in the space created by the swelling, or the creation of more pores after many CD cycles leading to a more accessible surface area and hence an increase in efficiency [32].

The CV curves at a scan rate of $20 \mathrm{mV} \mathrm{s}^{-1}$ before and after cycling are shown in Figure 6 (a). The cell showed an improved rate capability after 10, 000 cycles, which indicates that there was no decay in the capacitive performance of the cell, as shown in Figure 5 (d). Furthermore, no 
significant change to or degradation of the unique microstructure of the porous electrodes was revealed. In order to confirm the improved electrochemical performance of the cell after cycling, EIS measurements were also done before and after cycling (Figure 6 (b)). In general, at high frequencies, current flow in the cell is a result of the capacitance of the electrode materials. The intercept on the real axis (left side of the semicircle) of the Nyquist plot in the high-frequency region provides information about the combined resistance of the electrolyte, the contact leads and the porous carbon materials also known as the equivalent series resistance (ESR), denoted by $R_{S}$. The semicircle is a result of the charging of the double layer which appeared in the medium-frequency region. This is attributed to the interfacial charge transfer resistance and mass transport through the porous architecture of the material, and is denoted by $R_{\text {ст. }}$. The Nyquist plot shows a deviation from the vertical line in the low-frequency region, indicating a near-ideal capacitive behavior. This represents the diffusion of ions to the interface between electrode and electrolyte. At these low frequencies, the current flowing through the cell usually has resistance and a Warburg impedance characteristic element denoted by $W$, which is expressed as $/(j \omega)^{0.5}[33,34]$ where $A$ is the Warburg coefficient and $\omega$ is the angular frequency.

The relatively low value of the $R_{S}(0.29 \Omega)$ of the electrode material studied here reveals the fair conductivity and high quality of the 3D-PGP-1 electrodes. After 10,000 cycles there is a decrease in the semicircle indicating a decrease in the $R_{C T}$ value from 6.40 to $3.88 \Omega$. This observation is expected as EIS is a more sensitive technique, and it also confirms the results observed from the $\mathrm{CV}$ after cycling from the rectangular shape. 
a
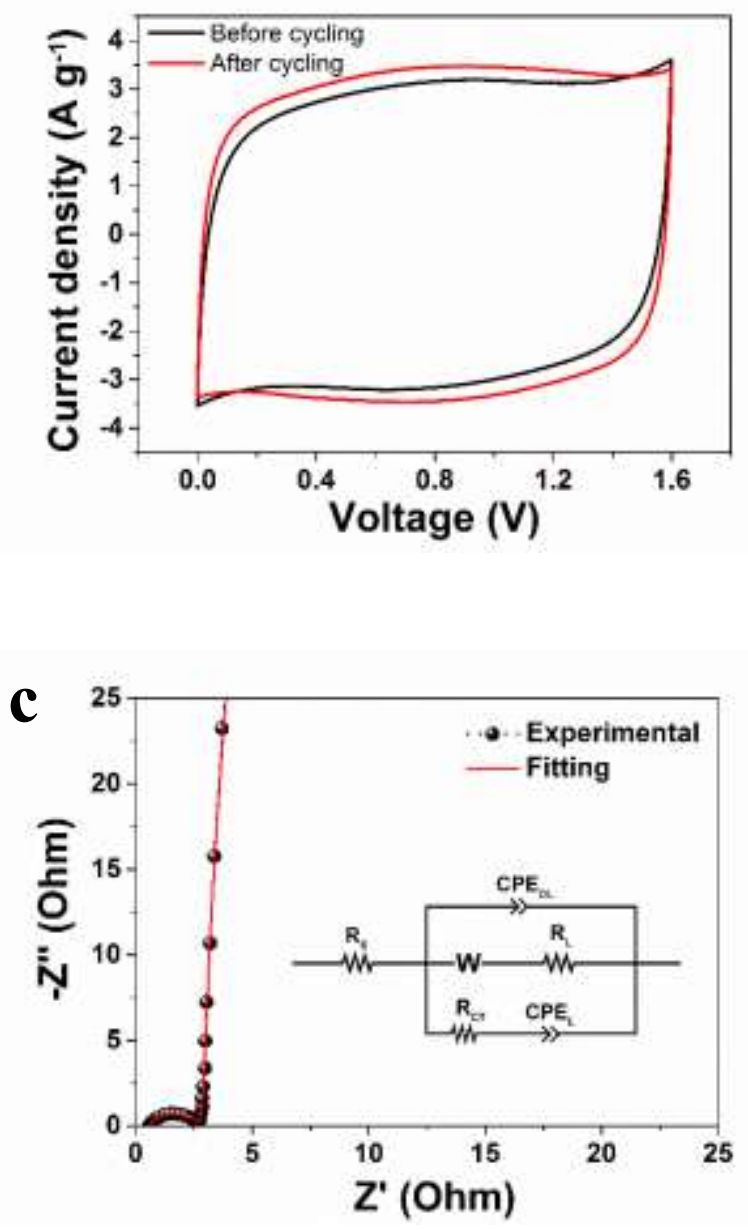

e

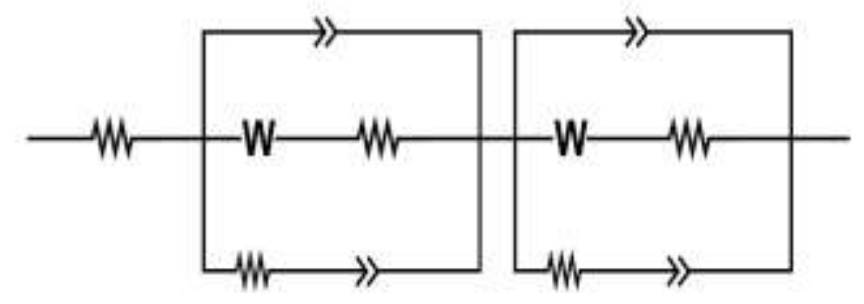

b
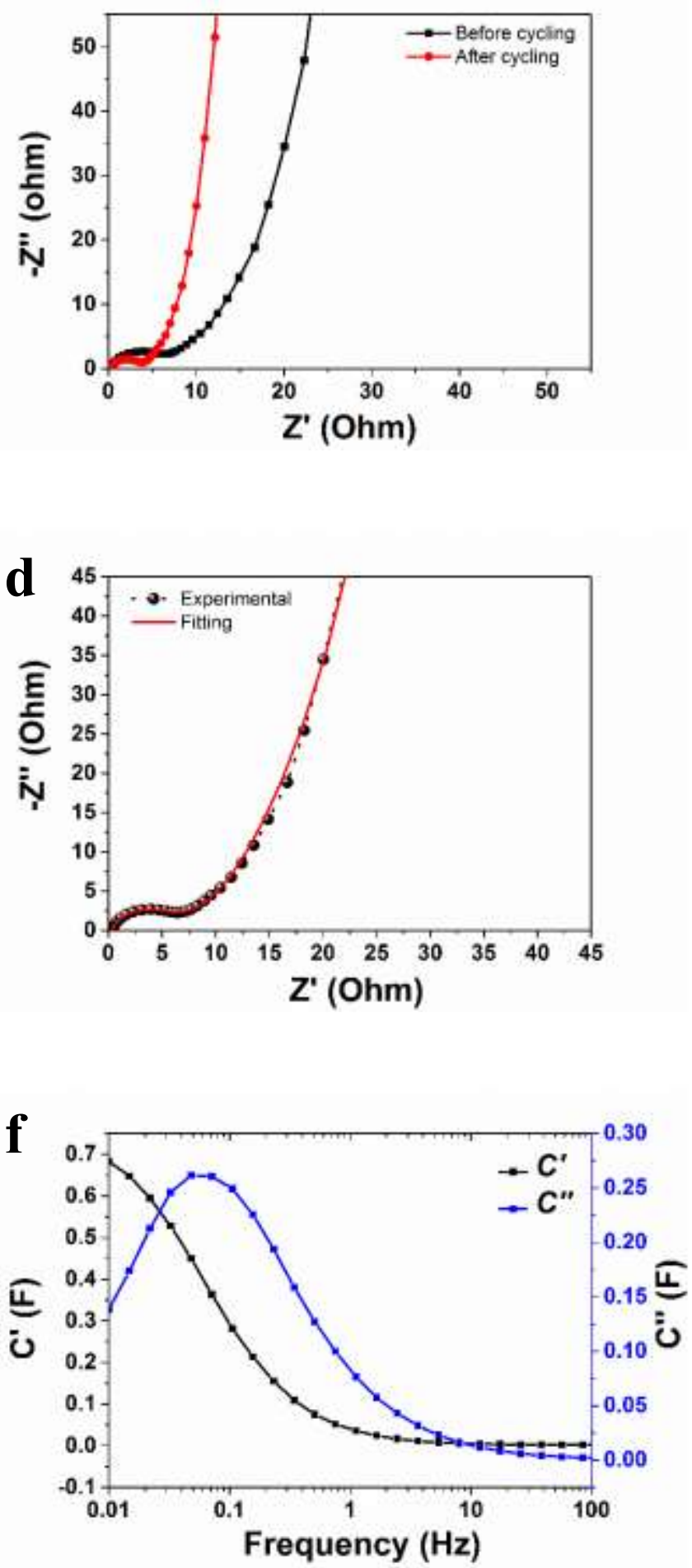

Figure 6. (a) CV curves at a scan rate of $20 \mathrm{mV}$ s-1 before and after cycling, (b) EIS before and after cycling, (c) EIS plot and fitting curve for single electrode with the inset to the figure being equivalent circuit used for fitting the data, (d) EIS plot and fitting curve for the symmetric cell, (e) Equivalent circuit used for fitting of the symmetric cell data in (d) and (f) the real and the imaginary part of the cells capacitance as a function of frequency. 
The capacitive behavior of the 3D-PGP-1 capacitor was also confirmed using impedance analysis. Figure 6 shows the Nyquist plot in the frequency range of $100 \mathrm{kHz}$ to $10 \mathrm{mHz}$ measured at the open circuit potential $(0 \mathrm{~V})$. The performance analyses based on Nyquist plot interpretations were represented and modelled by a modified Randles circuit [35] with a set of resistors and capacitors in series and parallel to extract the series and charge-transfer resistances. The fitting of the impedance expression data obtained from the experimental data of the Nyquist plot was performed with a fitting program ZFIT/EC-Lab version 10.38 and is represented in Figure 6 (c) along with the experimental data for a single electrode. In the equivalent circuit, a solution resistance $\left(R_{s}\right)$ is connected in series with a constant phase element $\left(C P E_{D L}\right)$, and the $C P E_{D L}$ is connected in parallel with the charge transfer resistance $\left(R_{C T}\right)$ and the mass capacitance $\left(C P E_{L}\right)$, while the Warburg and the leakage current resistance, $R_{L}$ are all in series with $R_{s}$. CPE electrode. $\mathrm{CPE}_{\mathrm{L}}$ represents the ideal polarizable capacitance which would give rise to a straight line parallel to the imaginary axis. The deviation from the ideal situation-suggests that a resistive element $R_{L}$ is associated with $C P E_{L}$. The impedance of $C P E_{D L}$ is defined as $Z_{C P E}=$ $\mathrm{T}(\mathrm{j} \omega)^{-\mathrm{n}}$ [34] where $\mathrm{T}$ and $\mathrm{n}$ are frequency-independent constants and $\omega$ is the angular frequency. The exponent $\mathrm{n}$ is a correction factor which is related to the capacitive kinetics and roughness of electrode surfaces. The values for $n$ range between 0 and $1 ; n=1$ denotes that the CPE is an ideal capacitor, for $n=-1$, CPE behaves as an inductor, while $n=0$ and 0.5 denote a resistance and Warburg behavior respectively [34].

Figure 6 (c) shows the Nyquist plot of the 3D-PGP-1 sample and its fitting using circuit in the inset to the figure obtained in a three-electrode configuration, while Figure 6 (d) shows the 
Nyquist plot and its fitting using the circuit in Figure 6 (e) of the symmetric cell of the 3D-PGP-1 sample. Both fittings show that the impedance spectroscopy of the electrodes fits perfectly with the experimental data. The inset to Figure 6 (c) shows the equivalent circuit of the single electrode, while Figure 6 (e) shows the equivalent circuit diagram of the symmetric cell by combining the two sets of fitting parameters listed in Table 1 for a single electrode in series. The $R_{S}$ and $R_{C T}$ values and other parameters fit perfectly without any further adjustment, which indicates an optimized minimization of experimental data with the design of the cell with an error of $0.1 . X^{2}$ represents the criterion for minimization of the fit. $X / \sqrt{\mathrm{N}}$, where $N$ is the number of points, is a normalized expression of $X^{2}$, whose value is independent of the number of points and is represented as the error above.

Table 1 Fitting parameters for the single electrode

\begin{tabular}{|c|c|c|c|c|c|c|c|}
\hline $\begin{array}{l}\mathbf{R}_{\mathrm{S}} \\
(\Omega)\end{array}$ & $\begin{array}{l}\mathrm{Q}_{\mathrm{DL}} \\
\left(\mathrm{F} . \mathrm{s}^{(\mathrm{a}-1)}\right)\end{array}$ & $a_{D L}$ & $\begin{array}{l}A_{W} \\
\left(\Omega . s^{-0.5}\right)\end{array}$ & $\begin{array}{l}R_{L} \\
(\Omega)\end{array}$ & $\begin{array}{l}\mathbf{R}_{\mathrm{CT}} \\
(\Omega)\end{array}$ & $\begin{array}{l}Q_{L} \\
\left(F . s^{(a-1)}\right)\end{array}$ & $a_{L}$ \\
\hline 0.300 & 0.0002 & 1 & 75.34 & 0.413 & 2.314 & 0.433 & 1 \\
\hline
\end{tabular}

The real and imaginary parts of the capacitance of the cell as a function of the frequency are shown in Figure 6 (f). $C^{\prime}$ represents the real accessible capacitance of the cell at the corresponding frequency $(0.01 \mathrm{~Hz})$, representing the deliverable capacitance which is $0.68 \mathrm{~F}$, while C" represents the energy loss due to the irreversible process of the electrodes. It is also used to define the transition frequency between pure capacitive and pure resistive behavior of 
the 3D-PGP-1 sample [36]. A small knee in the figure at a frequency of $0.1 \mathrm{~Hz}$ is an indication that the full capacitance of the cell can be reached at a slow recharging time $\tau=1 / \mathrm{f}$, which is 10 $\mathrm{s}$ in this case. This is an indication of the excellent and unique porous structures with a good distribution of micro- and mesopores which provide the highly accessible pathway to ions from the electrolyte. Figure $\mathrm{S} 4$ in the supporting information presents the phase angle as a function of frequency. The phase angle for the electrodes is about $\sim-80^{\circ}$, which is close to $-90^{\circ}$ and which defines ideal capacitive response.

\section{CONCLUSION}

In this work, we reported on the successful hydrothermal synthesis of three-dimensional porous carbons derived from various carbon precursors. The 3D material exhibited an interconnected carbon network with a superior surface area of $2,994 \mathrm{~m}^{2} \mathrm{~g}^{-1}$. The symmetric supercapacitor electrodes fabricated from these materials exhibited excellent electrochemical storage properties, including a high areal capacitance of $6.3 \mu \mathrm{F} \mathrm{cm}{ }^{-2}$, high energy of $0.56 \mu \mathrm{Wh}$ $\mathrm{cm}^{-2}\left(16.71 \mathrm{Wh} \mathrm{kg}^{-1}\right)$ and power densities of $13.39 \mu \mathrm{W} \mathrm{cm}-2\left(401 \mathrm{~W} \mathrm{~kg}^{-1}\right)$ with a stable operation voltage between 0 and $1.6 \mathrm{~V}$ and excellent long-term stability at a current density of $2 \mathrm{~A} \mathrm{~g}^{-1}$. The stable potential window in aqueous $\mathrm{KOH}$ electrolyte was attributed to the presence of functional groups in the porous carbon material which could prevent the over potential of dihydrogen and di-oxygen evolution in water-containing electrolyte. We believe that these interesting and promising results give the potential of these three-dimensional porous carbons the potential to be excellent electrode materials for high energy supercapacitors. 


\section{ACKNOWLEDGEMENT}

This work is based upon research supported by the South African Research Chairs Initiative (SARChi) in Carbon Technology and Materials of the Department of Science and Technology (DST) and the National Research Foundation (NRF). Any opinions, findings and conclusions or recommendations expressed in this work are those of authors, and the NRF and the DST do not accept any liability with regard thereto. F. Barzegar and D. Y. Momodu acknowledge financial support from the University of Pretoria and the NRF for their PhD bursaries. A. Bello and O.O. Fashedemi acknowledge the University of Pretoria's financial support for their Postdoctoral fellowship. Prof. Yury Gogotsi's group at Drexel University is thanked for the help in XPS measurements.

\section{REFERENCES}

[1] B.E. Conway, Electrochemical Supercapacitors: Scientific Fundamentals and Technological Applications, Kluwer Academic/Plenum, New York, 1999.

[2] E. Frackowiak, F. Béguin, F.F. Frackowiak, E., Beguin, Carbon materials for the electrochemical storage of energy in capacitors, Carbon 39 (2001) 937-950.

[3] J. Yan, Q. Wang, T. Wei, Z. Fan, Recent Advances in Design and Fabrication of Electrochemical Supercapacitors with High Energy Densities, Adv. Energy Mater. 4 (2014) $1-43$.

[4] M. Galiński, A. Lewandowski, I. Stępniak, lonic liquids as electrolytes, Electrochim. Acta. 51 (2006) 5567-5580.

[5] K. Fic, G. Lota, M. Meller, E. Frackowiak, Novel insight into neutral medium as electrolyte for high-voltage supercapacitors, Energy Environ. Sci. 5 (2012) 5842-5850.

[6] V. Khomenko, E. Raymundo-Piñero, F. Béguin, A new type of high energy asymmetric capacitor with nanoporous carbon electrodes in aqueous electrolyte, J. Power Sources. 195 (2010) 4234-4241. 
[7] D.-W. Wang, F. Li, M. Liu, G.Q. Lu, H.-M. Cheng, 3D aperiodic hierarchical porous graphitic carbon material for high-rate electrochemical capacitive energy storage, Angew. Chem. Int. Ed. Engl. 47 (2008) 373-376.

[8] C. Zhang, K.B. Hatzell, M. Boota, B. Dyatkin, M. Beidaghi, D. Long, et al., Highly porous carbon spheres for electrochemical capacitors and capacitive flowable suspension electrodes, Carbon 77 (2014) 155-164.

[9] B. You, L. Wang, N. Li, C. Zheng, Improving the Energy Storage Performance of Graphene through Insertion of Pristine CNTs and Ordered Mesoporous Carbon Coating, ChemElectroChem. 1 (2014) 772-778.

[10] B. You, P. Yin, J. Zhang, D. He, G. Chen, F. Kang, et al., Hydrogel-derived non-precious electrocatalysts for efficient oxygen reduction., Sci. Rep. 5 (2015) 11739.

[11] B. You, P. Yin, L. An, Multifunctional electroactive heteroatom-doped carbon aerogels., Small. 10 (2014) 4352-4361.

[12] J. Zhang, G. Chen, Q. Zhang, F. Kang, B. You, Self-Assembly Synthesis of N-Doped Carbon Aerogels for Supercapacitor and Electrocatalytic Oxygen Reduction., ACS Appl. Mater. Interfaces. 7 (2015) 12760-12766.

[13] N. Rajeswari, S. Selvasekarapandian, S. Karthikeyan, C. Sanjeeviraja, Y. Iwai, J. Kawamura, Structural, vibrational, thermal, and electrical properties of PVA/PVP biodegradable polymer blend electrolyte with $\mathrm{CH}_{3} \mathrm{COONH}_{4}$, lonics (Kiel). 19 (2013) 1105-1113.

[14] A.K. Swain, D. Bahadur, Enhanced Stability of Reduced Graphene Oxide Colloid Using Cross-Linking Polymers, J. Phys. Chem. C. 118 (2014) 9450-9457.

[15] D.J.T. Hill, A.K. Whittaker, Water diffusion into radiation crosslinked PVA-PVP network hydrogels, Radiat. Phys. Chem. 80 (2011) 213-218.

[16] R. Meena, R. Lehnen, B. Saake, Microwave-assisted synthesis of KC/Xylan/PVP-based blend hydrogel materials: physicochemical and rheological studies, Cellulose. 21 (2013) 553-568.

[17] A. Bello, O.O. Fashedemi, J.N. Lekitima, M. Fabiane, D. Dodoo-Arhin, K.I. Ozoemena, et al., High-performance symmetric electrochemical capacitor based on graphene foam and nanostructured manganese oxide, AIP Adv. 3 (2013) 082118.

[18] L. Zhang, F. Zhang, X. Yang, G. Long, Y. Wu, T. Zhang, et al., Porous 3D graphene-based bulk materials with exceptional high surface area and excellent conductivity for supercapacitors, Sci. Rep. 3 (2013) 1408. 
[19] Y. Zhu, S. Murali, M.D. Stoller, K.J. Ganesh, W. Cai, P.J. Ferreira, et al., Carbon-based supercapacitors produced by activation of graphene, Science. 332 (2011) 1537-1541.

[20] Y. Shi, D. Xiong, Microstructure and friction properties of PVA/PVP hydrogels for articular cartilage repair as function of polymerization degree and polymer concentration, Wear. 305 (2013) 280-285.

[21] Z. Ping, Q.T. Nguyen, J. Néel, Investigations of poly(vinyl alcohol)/poly(N-vinyl-2pyrrolidone) blends, 2 . Influence of the molecular weights of the polymer components on crystallization, Makromol Chem. 191 (1990) 185-198.

[22] Z. Chen, W. Ren, L. Gao, B. Liu, S. Pei, H.-M. Cheng, Three-dimensional flexible and conductive interconnected graphene networks grown by chemical vapour deposition., Nat. Mater. 10 (2011) 424-428.

[23] J. Wang, S. Kaskel, $\mathrm{KOH}$ activation of carbon-based materials for energy storage, J. Mater. Chem. 22 (2012) 23710-23725.

[24] M.P. Bichat, E. Raymundo-Piñero, F. Béguin, High voltage supercapacitor built with seaweed carbons in neutral aqueous electrolyte, Carbon 48 (2010) 4351-4361.

[25] Z.-S. Wu, W. Ren, D.-W. Wang, F. Li, B. Liu, H.-M. Cheng, High-energy MnO2 nanowire/graphene and graphene asymmetric electrochemical capacitors., ACS Nano. 4 (2010) 5835-5842.

[26] L. Demarconnay, E. Raymundo-Piñero, F. Béguin, A symmetric carbon/carbon supercapacitor operating at $1.6 \mathrm{~V}$ by using a neutral aqueous solution, Electrochem. Commun. 12 (2010) 1275-1278.

[27] H. Wang, Z. Xu, A. Kohandehghan, Z. Li, K. Cui, X. Tan, et al., Interconnected carbon nanosheets derived from hemp for ultrafast supercapacitors with high energy., ACS Nano. 7 (2013) 5131-5141.

[28] W. Tian, Q. Gao, Y. Tan, K. Yang, L. Zhu, C. Yang, et al., Bio-inspired beehive-like hierarchical nanoporous carbon derived from bamboo-based industrial by-product as a high performance supercapacitor electrode material, J. Mater. Chem. A. 3 (2015) 56565664.

[29] Y. Gogotsi, P. Simon, True performance metrics in electrochemical energy storage, Science. 334 (2011) 917-918.

[30] M. Zhi, F. Yang, F. Meng, M. Li, A. Manivannan, N. Wu, Effects of Pore Structure on Performance of An Activated-Carbon Supercapacitor Electrode Recycled from Scrap Waste Tires, ACS Sustain. Chem. Eng. 2 (2014) 1592-1598. 
[31] B. Xu, F. Wu, R. Chen, G. Cao, S. Chen, G. Wang, et al., Room temperature molten salt as electrolyte for carbon nanotube-based electric double layer capacitors, J. Power Sources. 158 (2006) 773-778.

[32] G. Ren, X. Pan, S. Bayne, Z. Fan, Kilohertz ultrafast electrochemical supercapacitors based on perpendicularly-oriented graphene grown inside of nickel foam, Carbon 71 (2014) 94-101.

[33] Y. Zhou, H. Xu, N. Lachman, M. Ghaffari, S. Wu, Y. Liu, et al., Advanced asymmetric supercapacitor based on conducting polymer and aligned carbon nanotubes with controlled nanomorphology, Nano Energy. 9 (2014) 176-185.

[34] H. Li, J. Wang, Q. Chu, Z. Wang, F. Zhang, S. Wang, Theoretical and experimental specific capacitance of polyaniline in sulfuric acid, J. Power Sources. 190 (2009) 578-586.

[35] C. Masarapu, H.F. Zeng, K.H. Hung, B. Wei, Effect of temperature on the capacitance of carbon nanotube supercapacitors., ACS Nano. 3 (2009) 2199-206.

[36] P.L. Taberna, P. Simon, J.-F.F. Fauvarque, Electrochemical Characteristics and Impedance Spectroscopy Studies of Carbon-Carbon Supercapacitors, J. Electrochem. Soc. 150 (2003) A292-300. 


\section{Supplementary Information}
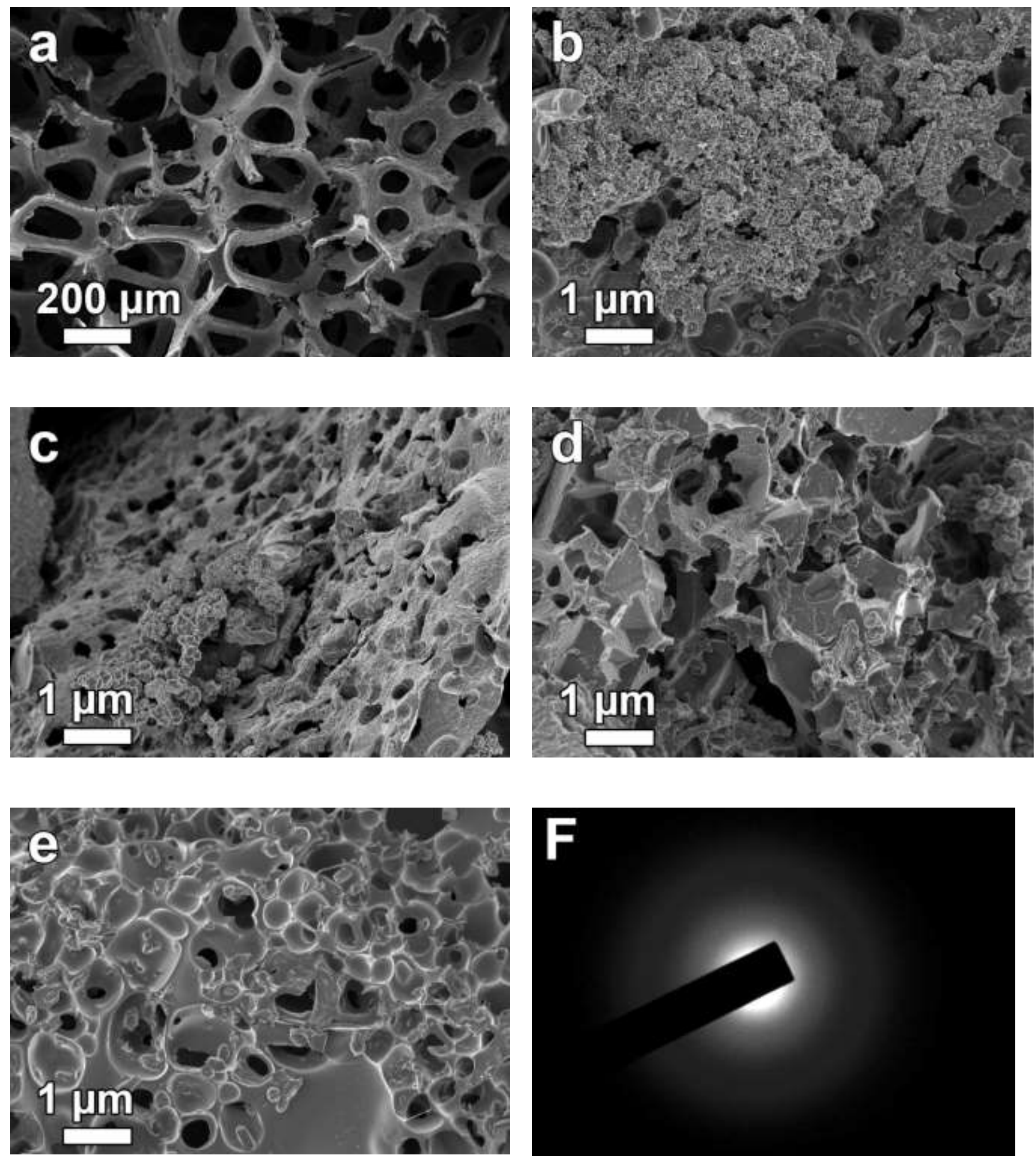

Supplementary figure S1. SEM images of (a) GF (b) 3D-PG-1 (c) 3D-PG-2 (d) 3D-PG-3 (e) 3D-PGP-2 (f) SAED image of 3D-PGP-1 
Supplementary Table S1. Elemental analysis of 3D-PGP-1

\begin{tabular}{|c|c|c|}
\hline C at.\% & N at.\% & O at.\% \\
\hline $89.4 \pm 0.1$ & $1.1 \pm 0.1$ & $9.5 \pm 0.1$ \\
\hline
\end{tabular}

Supplementary Table S2. XPS peak fitting results for 3D-PGP-1

\begin{tabular}{|c|c|c|c|c|c|}
\hline Region & BE [eV] & FWHM [eV] & Fraction & Assigned to & Reference \\
\hline & 284.7 & 0.9 & 0.45 & C-C & {$[1]$} \\
C 1s & 285.2 & 1.0 & 0.36 & $\mathrm{CH}_{\mathrm{x}}$ & {$[2]$} \\
& 286.2 & 1.5 & 0.16 & C-O-/C-N & [1] \\
\hline N 1s & 288.1 & 1.5 & 0.03 & COO & {$[1]$} \\
\hline O 1s & 400.6 & 2.1 & 1.00 & Nitrile/Pyrrole & {$[3]$} \\
\hline
\end{tabular}

The EDX below shows that the 3D-PGP-1 sample consist mainly of carbon with little or no traces of impurities. 


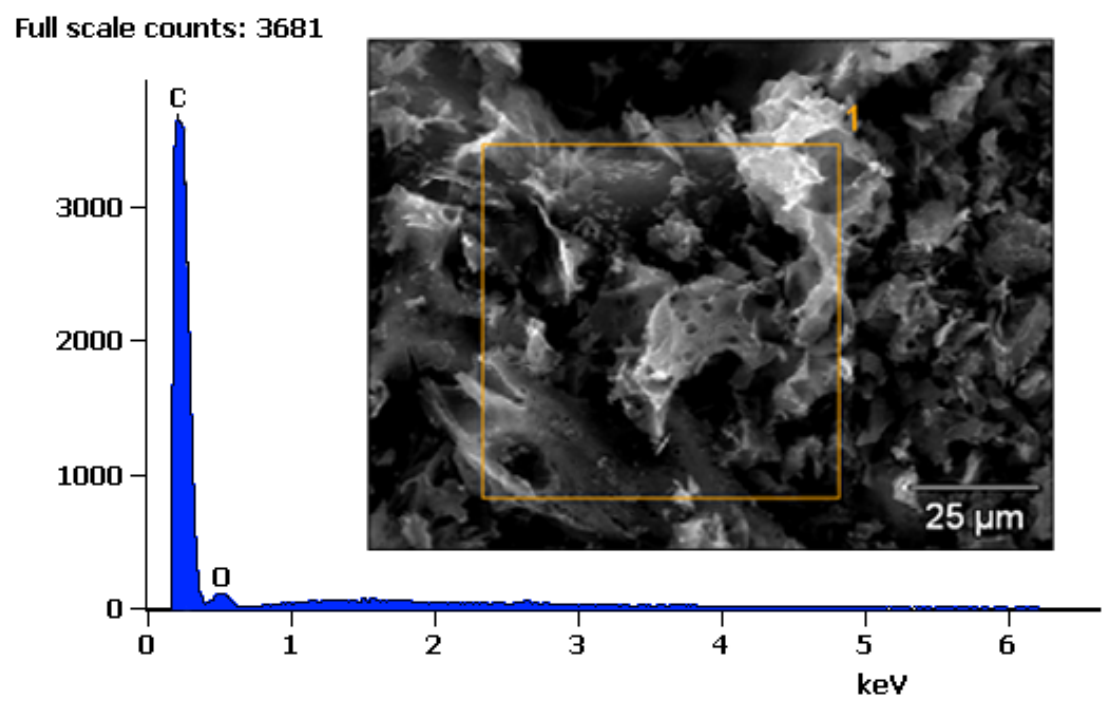

Supplementary figure S2. EDX pattern of 3D-PGP-1

The Raman spectra of the GF show the two prominent $G$ and 2D peaks at $1582.2 \mathrm{~cm}^{-1}$ and $2705.7 \mathrm{~cm}^{-1}$, respectively. The relative intensity $2 \mathrm{D} / \mathrm{G}$ ratio of 1.2 and $\mathrm{FWHM}$ of $38.8 \mathrm{~cm}^{-1}$ were attributed to few layer graphene. The absence of the D-peak (disorder) at $1350 \mathrm{~cm}^{-1}$ showed that the graphene foam was of good quality, with a low defect density. SEM shows the threedimensional (3D) porous network structure of the GF after CVD synthesis. 

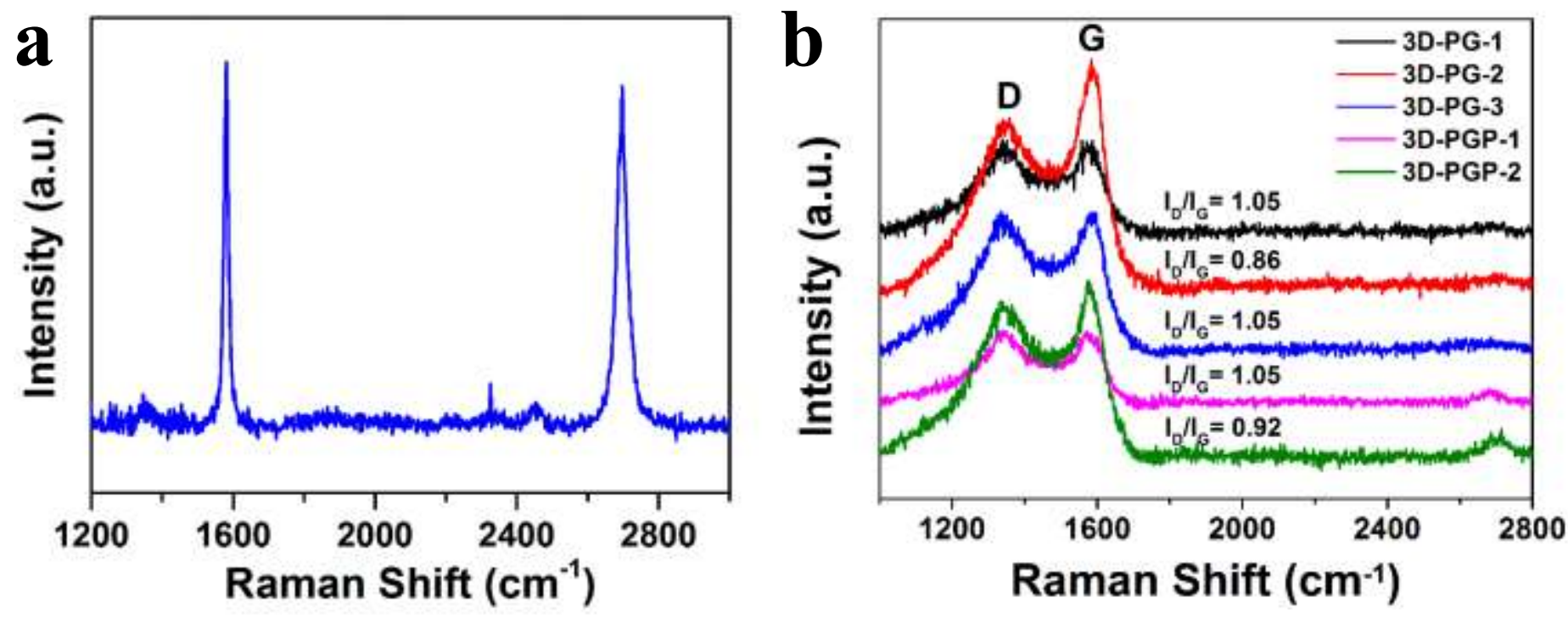

Supplementary figure S3. Raman spectra (a) GF and (b) 3D-PG-1, 3D-PG-2, 3D-PG-3, 3D-PGP-1, 3D-PGP-2.

These spectra were investigated using a Jobin Yvon Horiba TX 6400 micro-Raman spectrometer with a $514 \mathrm{~nm}$ argon excitation laser $(1.5 \mathrm{~mW}$ laser power on the sample to avoid possible thermal effects). The ratio of the $I_{D}$ and $I_{G}$ intensity peaks were used to approximate the degree of graphitization.

The structural properties such as BET and pore volume of the produced materials with different ration of GF is summarized in Table S1. BET was measured based on adsorption branch in the relative pressure range $\left(P / P_{0}\right)$ of 0.01 to 0.2 . 
Supplementary Table S3. BET and Pore volume of all the samples

\begin{tabular}{|c|c|c|}
\hline Sample & BET $\left(\mathbf{m}^{\mathbf{2}} \mathbf{~ g}^{-1}\right)$ & Pore volume $\left(\mathbf{c m}^{\mathbf{3}} \mathbf{g}^{-1}\right)$ \\
\hline 3D-PG-1 & 502 & 0.026548 \\
\hline 3D-PG-2 & 673 & 0.112935 \\
\hline 3D-PG-3 & 1063 & 0.131153 \\
\hline 3D-PGP-1 & & 0.455646 \\
\hline 3D-PGP-2 & 2994 & 0.248939 \\
\hline & & \\
\hline
\end{tabular}

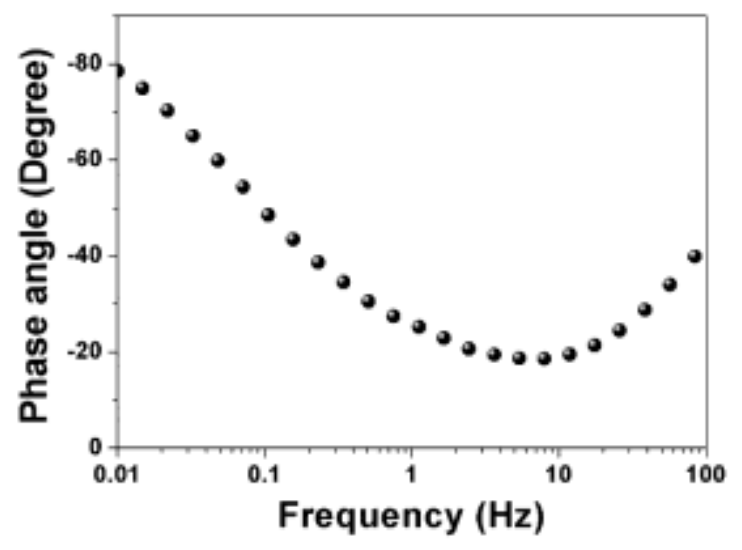

Supplementary figure S4. the phase angle versus frequency of the symmetric cell in $6 \mathrm{M} \mathrm{KOH}$ electrolyte. Showing a phase angle of $\sim-80^{\circ}$ which is close to $-90^{\circ}$ indicating a nearly ideal capacitive behaviour.

\section{References}

[1] A.P. Terzyk, The influence of activated carbon surface chemical composition on the adsorption of acetaminophen (paracetamol) in vitro, Colloids Surfaces A Physicochem. Eng. Asp. 177 (2001) 23-45.

[2] R.J.J. Jansen, H. van Bekkum, XPS of nitrogen-containing functional groups on activated carbon, Carbon N. Y. 33 (1995) 1021-1027.

[3] P.M. Jayaweera, E.L. Quah, H. Idriss, Photoreaction of Ethanol on TiO2(110) Single-Crystal Surface, J. Phys. Chem. C. 111 (2007) 1764-1769. 